\title{
Giant Gastric Folds in Juvenile Polyposis
}

\author{
Nicole B. Leonard Mary P. Bronner \\ Department of Pathology and ARUP Laboratories, University of Utah, Salt Lake City, UT, USA
}

\section{Keywords}

Juvenile polyposis syndrome · Ménétrier's disease - Giant gastric folds · Juvenile polyps .

Gastrointestinal pathology

\begin{abstract}
To advance the diagnostic accuracy of juvenile polyposis syndrome, an important yet often difficult diagnosis, we describe in detail a new and medically significant presentation. This hereditary and high-risk GI cancer syndrome is often associated with hereditary hemorrhagic telangiectasia, as in this 47-year-old female patient with a SMAD4 germline pathogenic mutation. Total gastrectomy revealed giant gastric folds with inflamed foveolar hyperplasia consuming most of the gastric cardia and body but sparing the antrum. Together, this gross and histologic pathology mimics Ménétrier's disease, an exceedingly rare and acquired protein-losing hypertrophic gastropathy. Classical gastric juvenile polyposis almost always and principally involves the antrum with multiple distinctive inflammatory polyps rather than the newly illustrated giant gastric folds of this case. No reports of giant gastric folds in juvenile polyposis have appeared in the literature. The distinction between juvenile polyposis and Ménétrier's disease is essential due to their disparate clinical outcomes and management. The differential considerations for giant gastric folds and inflamed gastric foveolar hyperplasia are fully reviewed. On the basis of this report, the differential for giant gastric folds must now expand to include juvenile polyposis syndrome. Genetic testing for pathogenic germline mutations of the 2 known causative genes of this syndrome, namely SMAD4 and BMPR1A, are readily available and should become part of the evaluation of giant gastric folds, particularly in view of the neoplastic and hereditary aspects of juvenile polyposis syndrome.
\end{abstract}

\section{Karger $\stackrel{2}{\circ}$}




\section{Introduction}

Juvenile polyposis syndrome is an uncommon autosomal dominant condition classically characterized by numerous juvenile polyps, also known as inflammatory polyps. It manifests throughout the gastrointestinal tract but predominantly involves the colon and stomach. The estimated incidence of the syndrome ranges between 1 in 16,000 and 1 in 100,000; however, the diagnosis is often difficult due to variable clinical presentations and overlap with other entities [1]. The 2 known causative genes for the syndrome are SMAD4 and BMPR1A, but only $50-60 \%$ of patients harbor an identifiable mutation [2]. The rarest reported manifestation of juvenile polyposis is massive gastric polyposis, which usually involves the antrum [3-5]. We present a patient with gastric juvenile polyposis syndrome with a pathogenic germline SMAD4 mutation who instead presented with giant gastric folds of the proximal stomach, rather than the characteristic gastric polyposis of the antrum. Pathologists should be aware of this gross and histologic variant of gastric juvenile "polyposis," which in this case was not a polyposis at all, but rather strongly mimicked the giant folds and antral sparing of Ménétrier's disease.

Giant gastric folds occur in numerous benign and malignant conditions of the stomach, including as a normal variant, due to proton pump inhibitor use, within hypertrophic lymphocytic gastritis, hypertrophic hypersecretory gastropathy, Zollinger-Ellison syndrome, tuberculosis, lymphoma, gastric adenocarcinoma, metastatic cancer, and even rare examples of gastrointestinal stromal tumors [6,7]. With this case report, hereditary juvenile polyposis syndrome must now be added to this important differential. The highly varied clinical consequences, neoplastic complications, and disparate management of these disorders make this an important though complex differential diagnosis in gastrointestinal disease.

\section{Case Report/Case Presentation}

This patient is a 47-year-old woman with a history of multiple monthly nosebleeds since childhood, intermittent mild anemia, and migraines throughout her life. Her first colonoscopy was performed at age 25 years for chronic constipation and abdominal pain. The medical records for her colonic findings are unavailable, but no specific concern for juvenile polyposis syndrome was raised at the time. No polyps were identified on subsequent upper endoscopy at the age of 28.

Germline genetic testing performed for an unrelated reason at age 45 revealed a pathogenic c.1245_1248delCAGA variant in the SMAD4 gene, leading to the diagnosis of juvenile polyposis syndrome with hereditary hemorrhagic telangiectasia. The patient's family history is pertinent for her father and 2 children, who also had histories of severe nosebleeds. Her son and daughter similarly suffered from migraines and her father had gastrointestinal polyps with severe gastrointestinal blood loss requiring transfusions.

Following diagnosis of juvenile polyposis syndrome in the patient and her family, the patient underwent additional colonoscopies at ages 45 and 46, revealing only 2 rectal hyperplastic polyps and one inflammatory cecal polyp. Multiple additional upper endoscopies; however, revealed strikingly enlarged gastric folds in the proximal stomach, involving $270^{\circ}$ around the circumference of the cardia and extending from the gastroesophageal junction into the greater curvature over a very large sessile area measuring over $5 \mathrm{~cm}$ in size. Extensive and repeated biopsies of the abnormal gastric mucosa revealed markedly inflamed gastric foveolar hyperplasia without Helicobacter pylori, intestinal metaplasia, dysplasia, or malignancy.

The large sessile area of massively enlarged gastric folds was not amenable to endoscopic resection. This and the high gastric cancer risk posed by juvenile polyposis, particularly in the setting of a SMAD4 germline mutation, lead to total gastrectomy. The resected specimen

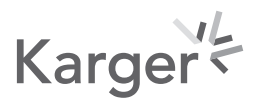


Fig. 1. Total gastrectomy demonstrating serpiginous giant gastric folds $\left({ }^{* * *}\right)$ occupying the majority of the gastric body, while grossly sparing the antrum (\#). The gastric fundus has a sessile mucosal thickening that is nearly circumferential ( + and + ) and extends to the GEJ, where a thin rim of pale gray squamous mucosa remains visible. The distal-most bowel resection shows an unremarkable antrum and cuff of duodenum (magnification, $\times 1$ ). GEJ, gastroesophageal junction.

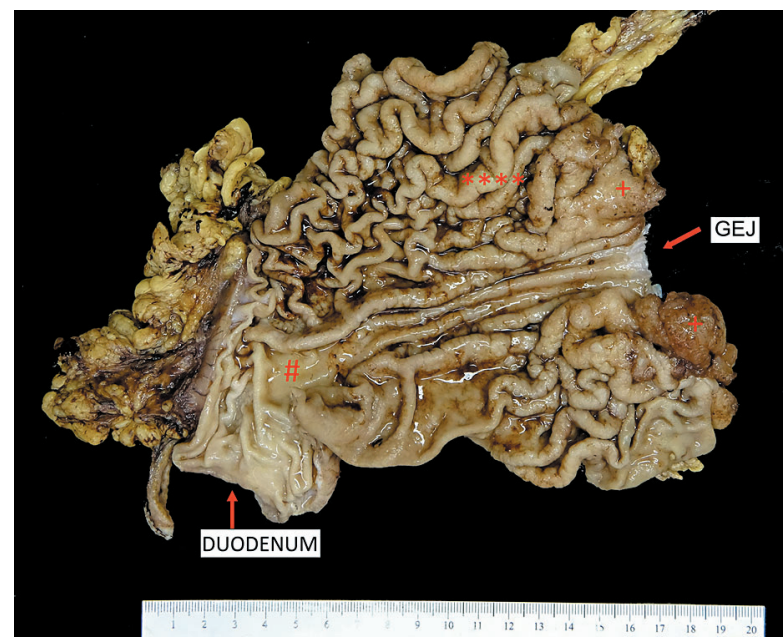

revealed a pink-red, massively thickened proximal gastric area of giant gastric mucosal folds, measuring overall $12.5 \times 9.0 \times 2.1 \mathrm{~cm}$. This altered mucosa extended from the gastroesophageal junction into the fundus and body of the stomach, encompassing an estimated $85 \%$ of the gastric luminal circumference (Fig. 1). Following extensive sampling of the gastrectomy, the lesional mucosa histologically showed massive foveolar hyperplasia with an edematous and inflamed lamia propria, and patchy cystically dilated glands, without intestinal metaplasia, dysplasia, or malignancy (Fig. 2, 3c). The giant gastric folds were composed histologically of elongated extensions of massively thickened mucosa with thin central cores of muscularis mucosae and scant submucosa. The mucosal thickening was largely due to massive hyperplasia of the surface or foveolar compartment of the mucosa, which made up an estimated $70 \%$ of the mucosal thickness throughout the region of giant folds. This was a marked increase over normal oxyntic mucosa where the foveolar surface makes up only $10-15 \%$ or less of the mucosal thickness (Fig. 3b). The oxyntic glands were normal (Fig. 3b). The distal specimen showed normal duodenal and gastric antral tissue, so that unlike most cases of gastric juvenile polyposis, the antrum in this patient's total gastrectomy was pathologically spared, both grossly and histologically (Fig. 1, 3a). SMAD4 immunohistochemistry was not performed as the patient's mutation was already known from sequencing. The most striking feature of this juvenile polyposis gastrectomy was the complete absence of classic polyposis, instead showing giant gastric folds of the cardia and body that spared the antrum. This work was approved by the University of Utah's institutional review board and informed patient consent and ethics approval was provided.

\section{Discussion/Conclusion}

This is the first known reported case of genetically, clinically, and pathologically documented juvenile polyposis syndrome with accompanying hereditary hemorrhagic telangiectasia, leading to proximal giant gastric folds rather than distal gastric polyposis. Interestingly, the patient lacked a significant colonic polyposis as well. Histologically, classical syndromic gastric juvenile polyps expand the gastric mucosal surface foveolar compartment with edema and inflammation. They commonly also show cystically dilated glands. In their essence, syndromic gastric juvenile polyps are histologically simply inflammatory-type polyps and as such are morphologically identical to the commonplace gastric hyperplastic polyp. Gastric 
Fig. 2. Classical juvenile polyp-type histologic changes with exuberant foveolar hyperplasia, an edematous and inflamed lamina propria, and patchy cystically dilated glands (a) (original magnification, $\times 100$ ). While polyps were grossly absent, and this is the novel hallmark of this report, the typical inflammatory features of juvenile polyposis are present histologically. Glandular dilation is exaggerated in an additional mucosal area (b) (original magnification, $\times 200$ ). Gastric foveolar epithelium reveals absence of dysplasia with normally sized, monolayered and basally oriented nuclei that lack pleomorphism (c) (original magnification, $\times 400)$.

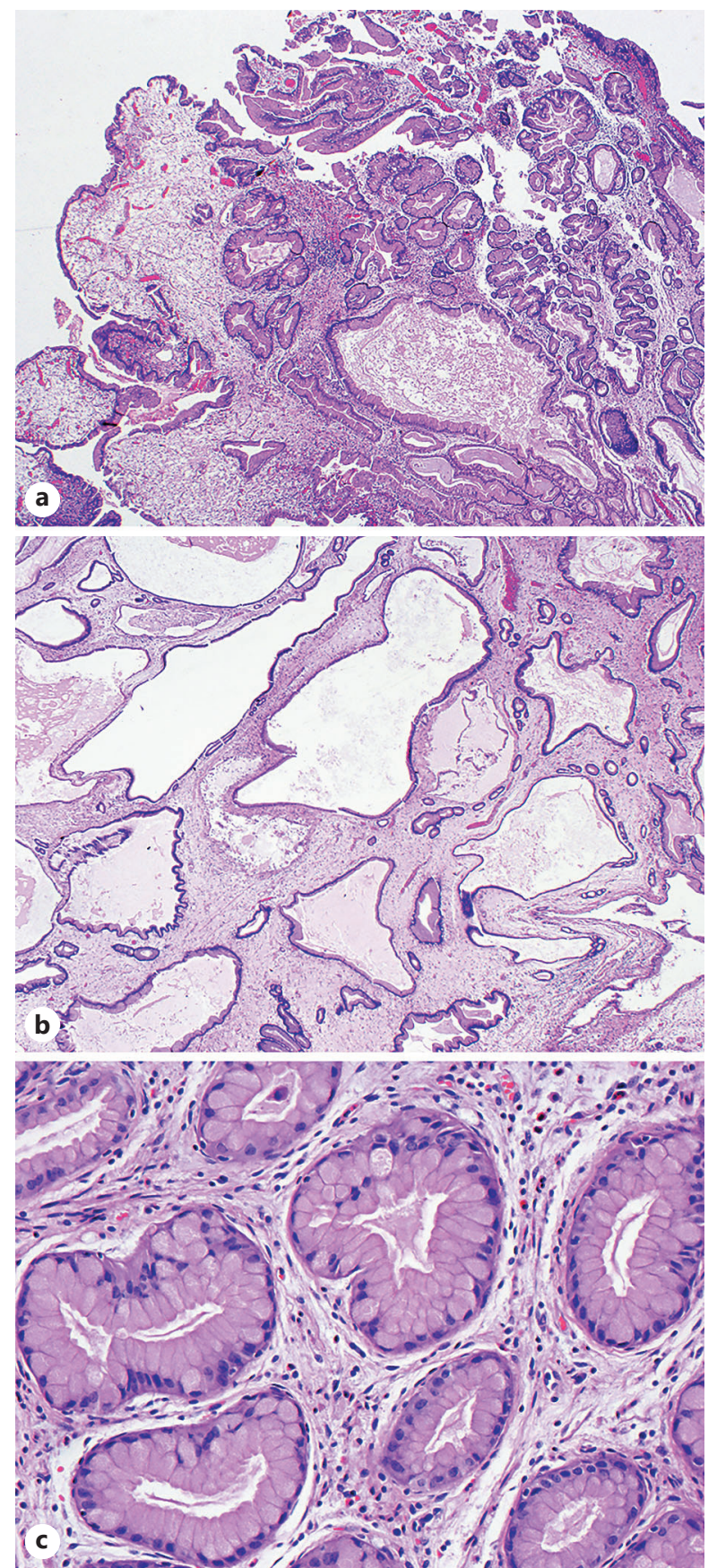

hyperplastic polyps are then the most common entity in the differential diagnosis $[3,5]$. Gastric hyperplastic polyps are frequent incidental findings and are benign in the overwhelming majority, although rare malignant transformation can occur [8]. A prior large series of incidental gastric hyperplastic polyps reveals an incidence of $1.79 \%$ of patients in the USA [9]. They are mostly solitary and antral and frequently arise in Helicobacter pylori or autoimmune gastritis $[8,9]$.

The specifically defined clinical diagnosis of juvenile polyposis syndrome requires 5 or more colonic juvenile/inflammatory polyps, juvenile/inflammatory polyps occurring throughout the gastrointestinal tract, or juvenile/inflammatory polyps arising in a family 
Fig. 3. The distal duodenal aspect of the total gastrectomy reveals normal duodenum with Brunner glands at the base of the duodenal mucosa (arrow) and normal antrum (arrow heads), with the typical antral 50/50 ratio of surface foveolar epithelium to deeper mucinous glands (a) (original magnification, $\times 100$ ). The normal antrum seen both on the gross image (Fig. 1) and here histologically is typical of Menetrier's disease and uncommon but present in this case of juvenile polyposis. The distal gastric body (b) (original magnification, $\times 100$ ) was also unremarkable histologically, showing the typical minor foveolar surface compartment (10-15\% or less) in contrast to the deeper oxyntic glandular compartment comprising the majority of the mucosal thickness. The proximal margin of the gastrectomy (c) (original magnification, $\times 100$ ), demonstrates massive foveolar hyperplasia (upper half) extending to the squamocolumnar gastroesophageal junction.

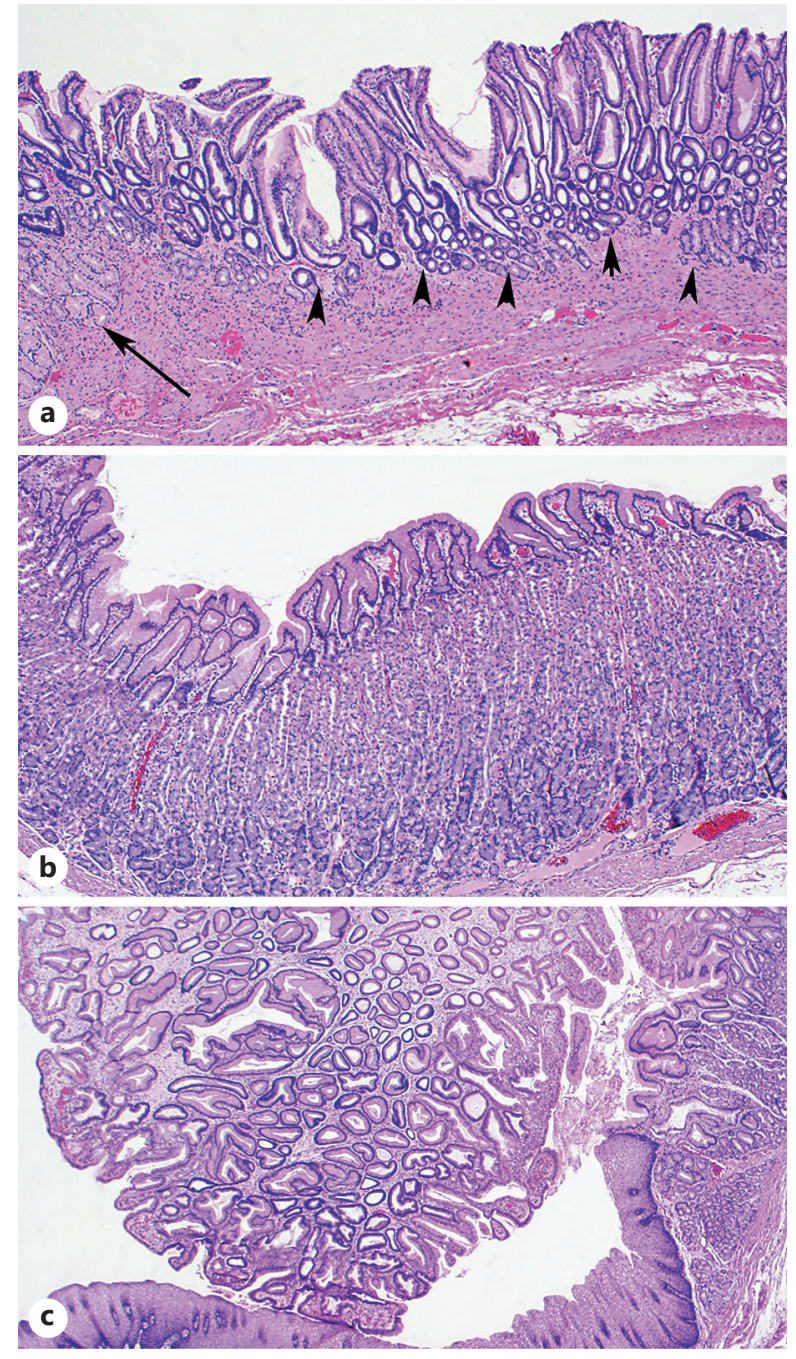

history of juvenile polyposis syndrome. The colonic focus of the initial criterion above is based on the predominantly colonic phenotype of this syndrome, which was not present in this case and is more common in patients with SMAD4 mutations. Anemia and gastrointestinal bleeding from the inflammatory polyps are common in syndromic patients. Many juvenile polyposis patients have undergone colectomy by the age of 20 in relation to severe gastrointestinal bleeding [5]. Patients with juvenile polyposis syndrome have an estimated $39 \%$ risk of colorectal cancer and a $21 \%$ risk of gastric cancer, warranting frequent endoscopic surveillance, polypectomy, and consideration of preventative gastrectomy and/or colectomy [3,5]. Pathogenic germline mutations in SMAD4 or BMPR1A help confirm the diagnosis $[3,5]$, but unfortunately, only $50-60 \%$ of juvenile polyposis patients have identifiable mutations [2]. It is possible, as with all inherited disorders, that this patient's germline SMAD4 pathogenic mutation was not completely penetrant, or that other genetic or environmental aspects may have influenced the phenotype presented. These could have included infectious, toxic, or other unknown elements individual to this patient.

Juvenile polyposis syndrome due to pathogenic germline SMAD4 mutation is more often associated with gastric polyposis and hereditary hemorrhagic telangiectasia [10], as in this case. Most patients with SMAD4 mutations have at least one clinical feature of hered- 
itary hemorrhagic telangiectasia, which may manifest as early as childhood, including epistaxis, telangiectasias, and arteriovenous malformations. These may result in the complications of anemia, migraine headaches, and exercise intolerance [1]. The risk of gastric cancer is also higher in patients with SMAD4 mutations than in those with BMPR1A mutations $[3,4]$. Patients with SMAD4 mutations have an estimated $30 \%$ risk of gastric cancer in comparison to the overall $11-21 \%$ risk of gastric cancer in all patients with juvenile polyposis syndrome $[4,11]$.

The American College of Gastroenterology recommends cancer screening for juvenile polyposis patients, to include colonoscopy and upper endoscopy every 1-3 years depending on polyp burden and beginning at the age of twelve or as early as symptoms occur [9]. Sayed et al. [12] suggest that those with SMAD4 mutations should be screened with upper endoscopy every 1-3 years, whereas those with BMPR1A mutations can be screened at longer 5-year intervals, based on the difference in gastric cancer risk from these 2 causative genes. Finally, patients with juvenile polyposis syndrome with hereditary hemorrhagic telangiectasia undergo surveillance not only for their gastrointestinal manifestations but also for the serious complications of hereditary hemorrhagic telangiectasia [1].

An essential aspect of the presented case is its mimicry of Ménétrier's disease. This disorder is thought to be acquired and results in a protein-losing hypertrophic gastropathy. It is far more rare than juvenile polyposis syndrome. Its precise incidence remains unknown due to its extreme infrequency. It is characterized by giant gastric folds usually sparing the antrum with massive and usually inflamed expansion of the gastric mucosal foveolar compartment, identical to the presentation of this case of SMAD4 juvenile polyposis. Ménétrier's patients present with similar symptoms as juvenile polyposis patients, including nausea, vomiting, diarrhea, abdominal pain, and weight loss, and edema due to hypoalbuminemia, as well as signs of malabsorption and protein loss [3, 5-7]. Gastric acid secretion is decreased and mucus production is increased due to massive foveolar hyperplasia and loss of oxyntic glands. Ménétrier's disease patients are more likely to have vomiting while those with juvenile polyposis are more likely to have anemia [7]. The patient presented here had a history of abdominal pain and intermittent mild anemia, but no other documented signs or symptoms of gastrointestinal polyposis or protein loss. Her serum albumin levels were normal. Finally, Ménétrier's is reportedly associated with an increased risk of gastric cancer, also parallel to gastric juvenile polyposis, but the exact risk is unknown due to its rarity $[6,7]$. This report suggests juvenile polyposis, with its well-established and high gastric cancer risk, as an important alternative consideration for patients diagnosed with Ménétrier's disease who do develop gastric cancer.

Histologically, the hyperplastic foveolar changes, edema, and inflammatory change of Ménétrier's disease are morphologically identical to those of juvenile polyposis and sporadic gastric hyperplastic polyps [6,7]. The oxyntic glands may become atrophic or cystically dilated in Ménétrier's disease. As with juvenile polyps, the lamina propria may be expanded by inflammation and edema to varying degrees and strands smooth muscle from the muscularis mucosae may involve the lamina propria, likely in relation to mucosal prolapse [6].

The exact pathogenesis of Ménétrier's disease is not well understood. A possible familial component has been reported [13], but this case report again suggests hereditary juvenile polyposis as an alternative consideration for suspected familial cases of Ménétrier's. Ménétrier's disease has occurred in patients with autoimmune disease, including inflammatory bowel disease, primary sclerosing cholangitis, and ankylosing spondylitis. Faster onset variants have been reported with infectious disorders such as cytomegalovirus gastritis, particularly in children, or those with Helicobacter pylori gastritis [6]. Ménétrier's disease is

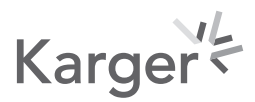


a classical clinicopathologic diagnosis requiring upper endoscopy, low gastric $\mathrm{pH}$, low serum albumin, normal serum gastrin, and full-thickness mucosal biopsies of involved mucosa [7]. On the basis of this case report of juvenile polyposis mimicking Ménétrier's disease, genetic testing may also be helpful in the evaluation whenever the exceedingly rare diagnosis of Ménétrier's disease is entertained.

While cases of juvenile polyposis syndrome presenting with massive gastric polyps have been reported, the presentation of giant gastric folds in the absence of polyps has not appeared in the previous literature of juvenile polyposis. The antrum is the most commonly involved gross gastric location in juvenile polyposis, but antral sparing has been reported in rare instances, including in 2 of 22 patients in the case series by Gonzalez et al. [4]. Composite review of 75 reported cases with gastric involvement from the literature, reveals only these 2 cases with antral sparing [3, 4, 14-24]. On the issue of antral involvement in Ménétrier's disease, Rich et al. [7] have reported several cases of Ménétrier's disease with an atypical antral involvement. Thus, sparing or involvement of the antrum is not an absolute feature of either disease [7], but antral sparing is highly characteristic of Ménétrier's and quite rare, but now reported, in this case of gastric juvenile polyposis. Knowledge of these variant gross disease distributions can nonetheless be very helpful in the differential diagnosis.

Here, we challenge the standard medical knowledge that antral sparing and gastric polyps versus giant gastric folds differentiate between gastric juvenile polyposis and Ménétrier's disease. This confirmed juvenile polyposis patient's illustrative pathology of giant gastric folds sparing the antrum to mimic Ménétrier's disease adds to our understanding that these variant manifestations do also occur in juvenile polyposis. Given the serious hereditary, malignant, and hemorrhagic complications of juvenile polyposis syndrome, this case provides support for SMAD4 and BMPR1A germline genetic testing [2], clinical evaluation, and a thorough family history before establishing the idiopathic diagnosis of Ménétrier's disease. In cases of giant gastric fold disease and inflamed foveolar hyperplasia of the stomach, awareness of the clinicopathologic overlap between gastric juvenile polyposis, Ménétrier's disease, and the wide range of additional causes, by pathologists, gastroenterologists, and oncologists alike, should aide in the correct diagnosis for these patients.

\section{Acknowledgments}

We sincerely thank our departed mentor, Doctor Rodger C. Haggitt, for his continuing inspiration to us to illuminate the pathology of the gastrointestinal tract, and in this particular instance, that of gastrointestinal polyposis syndromes, which formed one of his many fascinations.

\section{Statement of Ethics}

This work was approved by the University of Utah's institutional review board (IRB protocol \# 00046740) and written informed consent and ethics approval was obtained from the patient for publication of this report and any accompanying images.

\section{Conflict of Interest Statement}

The authors have no conflicts of interest to declare.

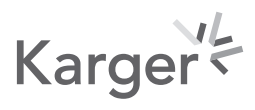




\section{Funding Sources}

This research did not receive any specific grant from funding agencies in the public, commercial, or not-for-profit sectors.

\section{Author Contributions}

M.P.B. and N.B.L. developed the study concept, design, methodology, writing and review of the paper, data acquisition, analysis, and interpretation, and provided all technical and material support. The authors read and approved the final paper.

\section{Data Availability Statement}

All data generated or analyzed during this study are included in this article. Further enquiries can be directed to the corresponding author.

\section{References}

1 Larsen Haidle J, Howe JR. Juvenile polyposis syndrome. In: Adam MP, Ardinger HH, Pagon RA, Wallace SE, Bean LJH, Mirzaa G, et al., editors. GeneReviews. Seattle: University of Washington; 1993-2020 [updated 2017 Mar 2017; Accessed 2021 Feb].

2 Brosens LA, Wood LD, Offerhaus GJ, Arnold CA, Lam-Himlin D, Giardiello FM, et al. Pathology and genetics of syndromic gastric polyps. Int J Surg Pathol. 2016;24:185-99.

3 Lawless ME, Toweill DL, Jewell KD, Jain D, Lamps L, Krasinskas AM, et al. Massive gastric juvenile polyposis: a clinicopathologic study using SMAD4 immunohistochemistry. Am J Clin Pathol. 2017;147:390.

4 Gonzalez RS, Adsay V, Graham RP, Shroff SG, Feely MM, Drage MG, et al. Massive gastric juvenile-type polyposis: a clinicopathological analysis of 22 cases. Histopathology. 2017 May;70:918-28.

5 Bronner MP. Gastrointestinal inherited polyposis syndromes. Mod Pathol. 2003;16:359-65.

6 Huh WJ, Coffey RJ, Washington MK. Ménétrier's disease: its mimickers and pathogenesis. J Pathol Transl Med. 2016;50:10-6.

7 Rich A, Toro TZ, Tanksley J, Fiske WH, Lind CD, Ayers GD, et al. Distinguishing Ménétrier's disease from its mimics. Gut. 2010;59:1617-24.

8 Abraham SC, Singh VK, Yardley JH, Wu TT. Hyperplastic polyps of the stomach: associations with histologic patterns of gastritis and gastric atrophy. Am J Surg Pathol. 2001;25:500-7.

9 Sonnenberg A, Genta RM. Prevalence of benign gastric polyps in a large pathology database. Dig Liver Dis. 2015;47:164-9.

10 Aretz S, Stienen D, Uhlhaas S, Stolte M, Entius MM, Loff S, et al. High proportion of large genomic deletions and a genotype phenotype update in 80 unrelated families with juvenile polyposis syndrome. J Med Genet. 2007; 44:702-9.

11 Syngal S, Brand RE, Church JM, Giardiello FM, Hampel HL, Burt RW. ACG clinical guideline: genetic testing and management of hereditary gastrointestinal cancer syndromes. Am J Gastroenterol. 2015;110:223-63.

12 Sayed MG, Ahmed AF, Ringold JR, Anderson ME, Bair JL, Mitros FA, et al. Germline SMAD4 or BMPR1A mutations and phenotype of juvenile polyposis. Ann Surg Oncol. 2002;9:901-6.

13 Larsen B, Tarp U, Kristensen E. Familial giant hypertrophic gastritis (Ménétrier's disease). Gut. 1987;28:151721.

14 Ma C, Giardiello FM, Montgomery EA. Upper tract juvenile polyps in juvenile polyposis patients: dysplasia and malignancy are associated with foveolar, intestinal, and pyloric differentiation. Am J Surg Pathol. 2014;38: 1618-26.

15 Hizawa K, lida M, Yao T, Aoyagi K, Fujishima M. Juvenile polyposis of the stomach: clinicopathological features and its malignant potential. J Clin Pathol. 1997;50:771-4.

16 Covarrubias DJ, Huprich JE. Best cases from the AFIP. Juvenile polyposis of the stomach. Radiographics. 2002; 22:415-20.

17 Papay KD, Falck VG, Poulsen SS, Panaccione R, Rehfeld JF, Storr MA. Juvenile polyposis of the stomach: a novel cause of hypergastrinemia. Nat Rev Gastroenterol Hepatol. 2010;7:583-8.

18 de Leon MP, Pedroni M, Viel A, Luppi C, Conigliaro R, Domati F, et al. Massive juvenile polyposis of the stomach in a family with SMAD4 gene mutation. Fam Cancer. 2019;18:165-72. 
19 Niv Y, Delpre G, Sperber AD, Sandbank J, Zirkin H. Hyperplastic gastric polyposis, hypergastrinaemia and colorectal neoplasia: a description of four cases. Eur J Gastroenterol Hepatol. 2003;15:1361-6.

20 Pintiliciuc OG, Heresbach D, de-Lajarte-Thirouard AS, Dugast C, Reignier A, Cottereau J, et al. Gastric involvement in juvenile polyposis associated with germline SMAD4 mutations: an entity characterized by a mixed hypertrophic and polypoid gastropathy. Gastroenterol Clin Biol. 2008;32:445-50.

21 Kelly S, Dwerryhouse S, Safranek P, Hardwick R. Juvenile polyposis syndrome affecting the stomach: a case report. J Med Case Rep. 2008;2:314.

22 Wong-Chong N, Kidanewold WH, Kirsch R, May GR, McCart JA. Giant stomach secondary to juvenile polyposis syndrome. J Gastrointest Surg. 2012;16:669-72.

23 Stadler ZK, Salo-Mullen E, Zhang L, Shia J, Bacares R, Power DG, et al. Juvenile polyposis syndrome presenting with familial gastric cancer and massive gastric polyposis. J Clin Oncol. 2012;30:e229-32.

24 Soer E, de Vos Tot Nederveen Cappel WH, Ligtenberg MJ, Moll F, Pierik RG, Vecht J, et al. Massive gastric polyposis associated with a germline SMAD4 gene mutation. Fam Cancer. 2015;14:569-73. 